\title{
Two Systems of Remnant Movement II and Extraction from Specifier Position
}

\author{
Craig Thiersch \\ University of Potsdam and University of Tilburg
}

\section{Introduction}

In the wake of Kayne's Antisymmetry Hypothesis and Linear Correspondence Axiom (LCA), there has been much fruitful research attempting to adjust syntactic analyses to those permitted by Kayne's restrictive system. In doing so, analyses which at first seem counter-intuitive may turn out to provide solutions to old problems. Two cases in point are the analysis of Malagasy involving extensive Remnant Movement [henceforth RM] described in Rackowski \& Travis (2000), Pearson (2001), and elsewhere; on the one hand, and the analysis of Hungarian and Dutch verbal clusters in Koopman \& Szabolcsi (2000) [henceforth R\&T, Pearson, and K\&Sz]. ${ }^{1}$

The original motivation (in part) for examining L\&Sz and subsequently $R \& T$ was that it is the extensive use of iterated RM which increases the computational complexity of languages generatable in Stabler's "Strict Minimalist Grammar" formalism over that of context-free grammars. It has also been noted that allowing extraction from complex specifiers created by Merge (as opposed to Move) increases the level of complexity even further (Jens Michaelis, p.c.). Both R\&T and $\mathrm{K} \& S z$ make extensive use of RM; R\&T allow extraction from complex specifiers, while $\mathrm{K} \& \mathrm{Sz}$ do not. Although the specifiers in both cases are created by Move, not Merge, we nevertheless feel that there is enough intrinsic linguistic interest in trying to limit extraction possibilities to pursue the comparison of these two systems with regard to this point. Cf. Thiersch (in prep.b) for discussion. ${ }^{2}$

This is intuitively plausible, as we know extraction from deeply embedded position within specifiers is usually ungrammatical:

(1) a. $\mathrm{Who}_{i}$ did Fred say that Susan hoped for Sam to kiss $e_{i}$ ?

b. * $\mathrm{Who}_{i}$ did Fred say that for Sam to kiss $e_{i}$ would create a scandal ?

As noted above extraction from complex specifiers is explicitly forbidden in $\mathrm{K} \& \mathrm{Sz}$ and the prohibition forms a crucial part of their analysis. It appears however to be necessary for R\&T. In this article we compare the two systems in the hope of shedding some light of how two rather different systems involving RM have coped with the same problem: the position of nominal objects.

As a terminological matter we distinguish between two types of RM, I and II, following Müller (2002) and Thiersch (2002):

(2) RM I

a. Erschossen hat er Bin Ladin schon gestern;

b. Analysis: [ ${ }_{\mathrm{vP}} e_{i}$ erschossen $]_{j}$ hat er [Bin Ladin $]_{i}$ schon gestern $e_{j}$

(3) RM II

a. John reads no novels
b. John [reads $\left.e_{i}\right]_{j}\left[\alpha[\text { no novels }]_{i} e_{j}\right]$ 
Although formally identical, in that they involve displacing a constituent $\alpha$ from which some element $\beta$ has been extracted so that the antecedent, $\beta$, no longer C-commands its trace in $\alpha$, they tend to have different characteristics. For example, in RM I both operations exist as independent constructions in the language (in (2) "scrambling" and Vorfeld-topicalization), and it cannot be iterated; RM II generally can apply repeatedly, and as (3b) shows, neither operation occurs independently, and its effects are often invisible - except for semantic effects such as the two readings of (4a); cf. Kayne (1998). ${ }^{3}$

(4) a. They forced him to marry no one

b. They forced him [[to marry $\left.\left.e_{i}\right]_{j}[\text { no one }]_{i} e_{j}\right]$

c. They [forced him to marry $\left.e_{i}\right]_{j}$ [no one $]_{i} e_{j}$

Before we examine the problem relating to the position of nominal DPs, we briefly sketch some relevant aspects of Malagasy syntax and three sample empirical problems the RM analysis apparently solves. For a more detailed account of Malagasy syntax the reader is referred to Pearson (2001) and the references therein.

\section{The basics: the Malagasy voicing system}

Roughly, Malagasy is a verb initial language (although there are constructions in which certain "fronted" constituents may precede the verb). It is sometimes cited as being typologically VOS, but this oversimplifies what is really going on. Basically the arguments line up following the verb, and depending on the voice of the verb, one of them is "promoted" to the right most position. Schematically,

(5) $\mathrm{V}_{\text {voice2 }} \arg _{1}\left(\underset{\arg _{2}}{ }\right) \arg _{3} \underset{\arg _{2}}{\arg _{2}}$

That is, if the verb is in "second" voice, the second argument appears to the right. ${ }^{4}$ The five voices are traditionally called Nominative, Accusative, Dative, Transitive, Circumstantial). Cf. Pearson (2001), Chap.2 [his (65)]:

(6) a. Mamono akoho amin'ny antsy ny mpamboly

NomP.kill chicken with-Det knife Det farmer

'The farmer kills chickens with the knife'

b. Vonoin'ny mpamboly amin'ny antsy ny akoho AccP.kill-Det farmer with-Det knife Det chicken

'The chickens are killed by the farmer with the knife' or 'The chickens, the farmer is killing (them) with the knife'

c. Amonoan'ny mpamboly akoho ny antsy CrcP.kill-Det farmer chicken Det knife 'The knife is being used by the farmer to kill chickens' or 'The knife, the farmer is killing chickens (with it)'

Indeed, the voicing system has sometimes been compared with Indo-European passive, and the right-most argument called the subject, although the construction has rather different properties, as can be seen in (6b), the non-promoted semantic subject does not become an optional chômer in a PP, but remains, presumably in situ. ${ }^{5}$ Earlier analyses often assumed that the structure was "right 
branching"; cf. Guilfoyle, Hung \& Travis (1992). That is, the external argument moved up to a right-hand specifier position. Pearson (and R\&T) argue that this is not the case, but that the basic structure is consistantly Spec-HeadComp, as in Kayne's antisymmetry proposal, and that the external argument has moved up to the left, and a constituent containing the verb with the remaining arguments (and some adjuncts) has then moved leftward around the externalized argument. Schematically, the sentence in (6b) would have a structure roughly like

(7) [Vonoin'ny mpamboly $e_{i}$ amin'ny antsy $]_{j}[\text { ny akoho }]_{i} e_{j}$ AccP.kill-Det farmer e.c. with-Det knife Det chicken e.c.

This is grossly over-simplified and ignores the functional categories as well as the considerable difference in hypothesized nodes between, say, Pearson and R\&T, but captures the spirit of the analyses under consideration. Pearson in particular motivates a number of functional categories and hence movements, giving a more complete and differentiated account of the facts.

We briefly summarize in the following sections how this type of analysis accounts for some empirical observations, indicating that the RM approach is on the right track, before turning to the problem of placing the DP arguments.

\section{Some empirical problems solved}

Focus "extraction": Various focus and topic particles can allow an element to be "extracted" to the left, but as has been long noted in the literature, this can only be the constituent which would have been "externalized" to the rightmost position according to the voice of the verb. ${ }^{6}$ E.g., [Pearson, Chap.2 (36)]:
a. * Ny akoho no namono
tamin'ny antsy ny
Det chicken Foc Pst-NomP.kill Pst-with-Det knife Det
mpamboly
farmer
'It's the chicken that the farmer killed with the knife'
b. Ny akoho no novonoin'ny mpamboly tamin'ny Det chicken Foc Pst-AccP.kill-Det farmer Pst-with-Det antsy knife
c. * Ny akoho no namonoan'ny mpamboly ny antsy Det chicken Foc Pst-CrcP.kill-Det farmer Det knife

This is surprising under the Guilfoyle, Hung \& Travis analysis, as one generally expects objects to be more easily extractible than subjects; cf. Sabel (2003) for discussion and an alternative to the RM analysis. ${ }^{7}$ In a structure like (7), however, the external object is on the main rightward projection line with no barrier-like categories in inbetween, whereas the other arguments are embedded in the fronted TP/VP, i.e. a complex specifier and presumably an island (a point to which we return below).

Placement of discourse markers: Pearson discusses another phenomenon (noted in passing in R\&T), namely that various discourse markers come in peculiar places and don't seem to have any particular pattern. For example, the yes/no 
question marker ve appears in the penultimate position (left of the externalized argument) in ordinary sentences, but in the second position (between the fronted DP and the particle no!) in focus sentences [his Chap.2: (14a) \& Chap.4: (102a)]:

(9) a. Vonoin'ny mpamboly amin'ny antsy ve ny akoho?

AccP.kill-Det farmer with-Det knife Qu Det chicken

'The chickens, is the farmer killing (them) with the knife?'

b. I Bakoly ve no manapaka bozaka?

Det Bakoly Qu Foc NomP.cut grass

'Is it Bakoly who is cutting the grass?'

Pearson points out that we only need to assume that $v e$ is a second-position functional head which raises the specifier of its complement in order to account for these facts; in (9a) the whole TP/IP is raised, leaving the externalized argument to the right of ve. in (9b), however, only the focused DP is raised (see the structure in footnote 7).

Adverb order: Finally we come to one of the phenomena which forms the centerpiece of the R\&T article, namely the order of adverbials. The basic assumption is the universal order of adverbials related to a hypothesized universal heirarchy of functional projections proposed in Cinque (1999). Language specific deviations were to be accounted for, as usual, by language particular properties (e.g., lexical). As previously discussed with a similar analysis in Rackowski (1998), the order of adverbials in Malagasy deviates from the proposed universal order in a surprising way; aside from some minor peculiarities, the pre-verbal adverbs mimic the Cinque order, whereas the post-verbal adverbs are in the mirror-image order:

a. Cinque's order:

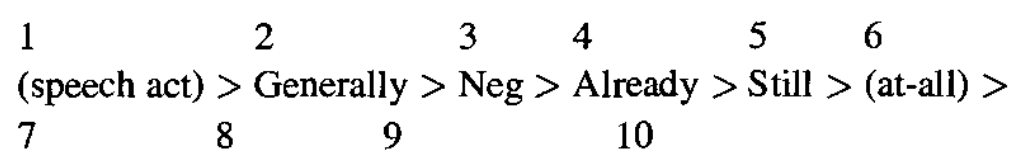

Anymore $>$ Always $>$ Completely $>$ Well

b. Malagasy order:

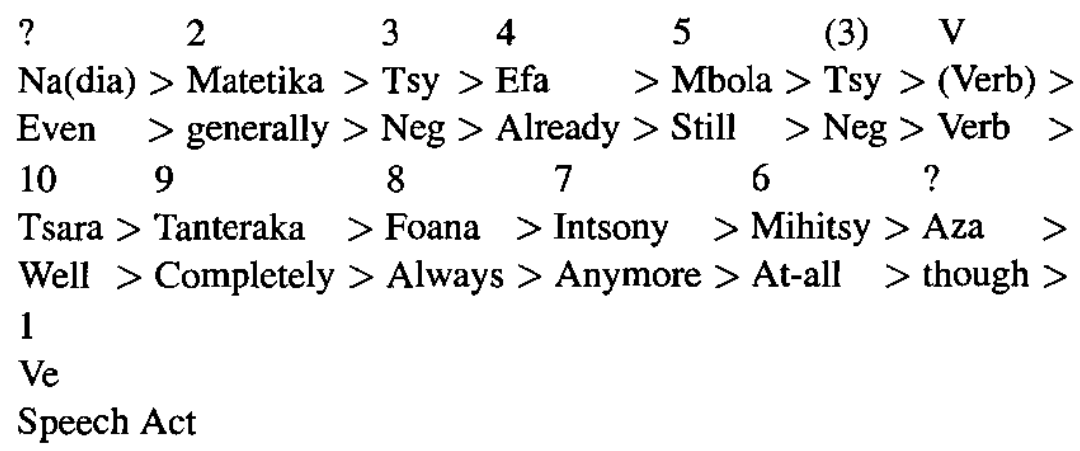

This immediately suggests a "roll-up" operation like the operation proposed in $\mathrm{K} \& \mathrm{Sz}$ to reverse the order of Hungarian verbs. The language particular stipulation they need to make is that the upper adverbs (up to and including Neg "tsy") are generated in Spec of their functional projections; the lower adverbs (6-10) generated as heads of their projections ${ }^{8}$ Their suggested derivation works as follows: 
(11) a. Repeated movement to $S p e c, A d v_{x} \mathrm{P}$ (roll-up) reverses the order,

b. a blocking trigger (e.g., an element in Spec,NegP) stops the roll-up, and

c. a high resulting constituent (e.g., TP) is moved to a projection above the external argument (subject).

The following tree illustrates (11a-b), step (11c) was illustrated (for a sentence without adverbs) in (7) [cf. their (12)-(13), p.122]:

(12) a. Tsy manasa tsara tanteraka foana intsony ny lamba mihitsy not wash wel completely always anymore the clothes at-all

Rakoto

Rakoto

b.

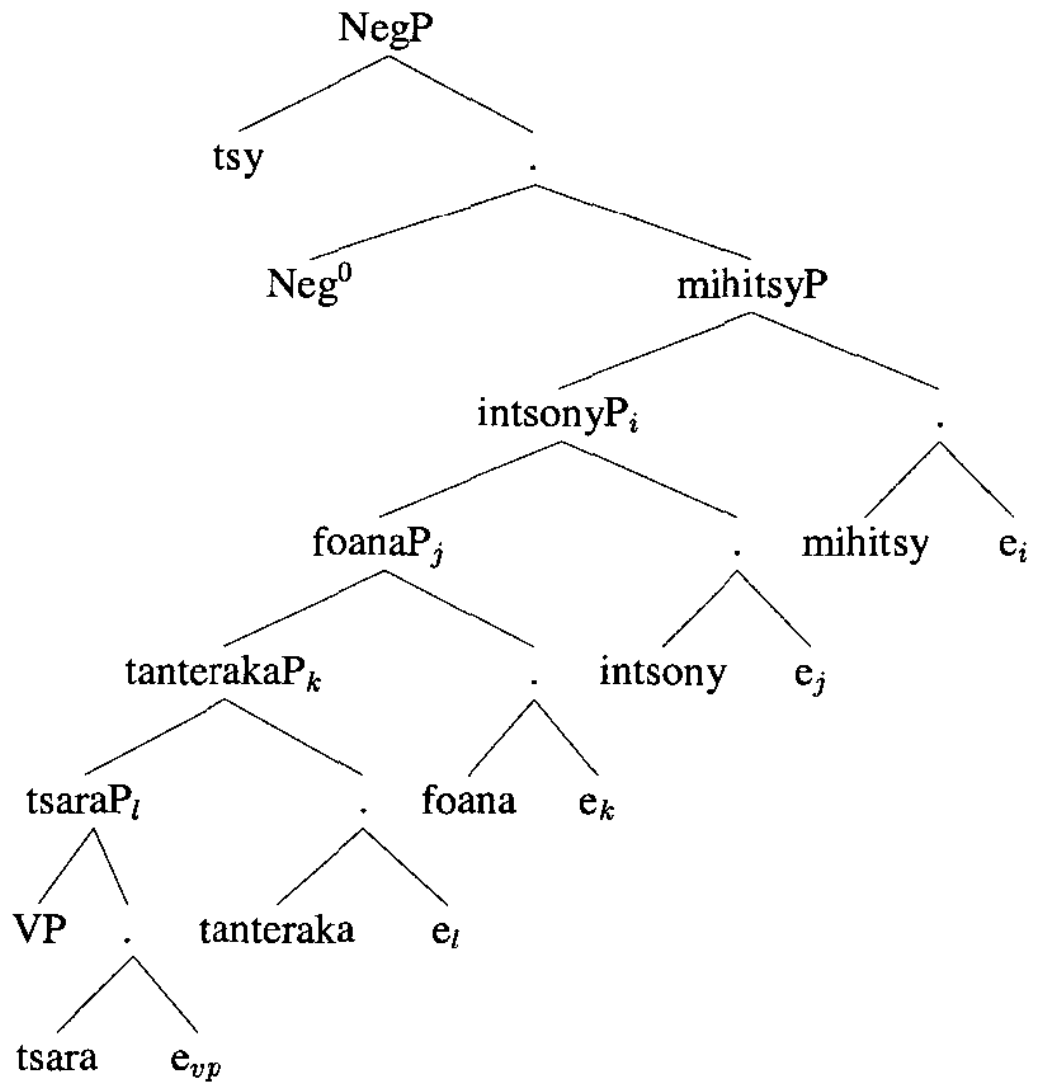

Note that the tree in (12b) already involves repeated RM, even without DP arguments. While one can quibble with many of the details and assumptions, the capturing of these three phenomena (other arguments are given in Pearson and $R \& T$ ) seems sufficient motivation that the $R M$ approach is on the right track, and we turn to the details of DP placement.

\section{The order of DP objects}

The problem is illustrated by two of their examples. Definite direct objects may "optionally appear among or after postverbal adverbials." [p.125] This is illustrated in (13) [their $(21 \mathrm{a} / \mathrm{b})]^{9}$ :

a. Tsy manasa

foana ny lamba mihitsy Rakoto.

NEG PRES.AT.wash always det cloths at-all $R$.

'Rakoto does not always wash the clothes at all' 


\section{b. Tsy manasa intsony mihitsy ny lamba Rakoto. NEG PRES.AT.wash no-longer at-all det cloths $R$. \\ 'Rakoto does not wash the clothes at all any more'}

The adverbs mihitsy and foana are respectively Adv6 and Adv8 in the Cinque heirarchy, here appearing in $8>6$ order due to the (remnant) movement of the verb phrase. How would the derivation procede? The first merge would be with the direct object, yielding

$$
\text { [VP manasa [DP ny lamba]] }
$$

This is merged with the lower adverb, foana, and the VP moves to the specifier of foana, yielding

$$
\text { [Adv8P [VP }{ }_{\mathrm{VP}_{i}} \text { manasa [DP ny lamba]] foana } e_{i} \text { ] }
$$

If we simply merge again and move the Adv8P out, we get

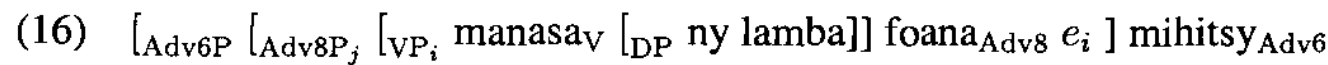
$\left.e_{j}\right]$

This generates neither (13a) or (13b), but is grammatical. ${ }^{10}$ Supposing we had immediately extracted the DP to Spec,AgrOP yielding

$$
\text { [AgrOP }\left[\mathrm{DP}_{i} \text { ny lamba] }\left[\mathrm{AgrO}^{\prime} \mathrm{AgrO}^{0}\left[\mathrm{VP} \text { manasa } e_{i}\right]\right]\right]
$$

Merging with Adv6P and raising the remnant gives (18a) shown as tree in (18b):

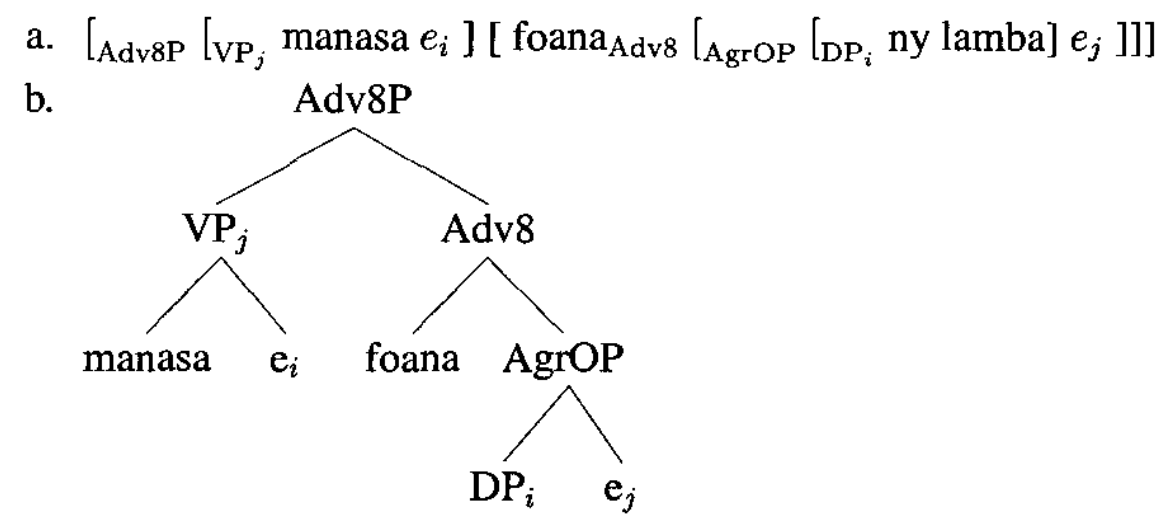

And after merging with Adv6 and raising Adv8P, we get the structure for (13a):

$$
\begin{aligned}
& {\left[\operatorname { A d v 6 P } _ { \mathrm { P } } \left[\operatorname { A d v 8 P } _ { k } \left[\mathrm{VP}_{j}\right.\right.\right.} \\
& \text { mihitsy } \left._{\mathrm{Adv}} e_{k}\right]
\end{aligned}
$$

What about (13b)? Here there at least two possibilities, each with attendant problems. One possibility is to extract the DP immediately, as in the derivation for (13a). But then we would need to extract it again to get (13b). However, we have already "used up" AgrOP and checked the appropriate feature. This means we would have to postulate another functional category. This has the disadvantage of needing to postulate a potentially unlimited number of functional categories (and features) just to effect the extraction. (This is in fact the solution adopted by K\&Sz; see below.)

The alternative, adopted by R\&T, is to take the DP along, as in (16), and wait until the appropriate moment in the derivation, then merge once with AgrOP and extract the DP: 
(20)

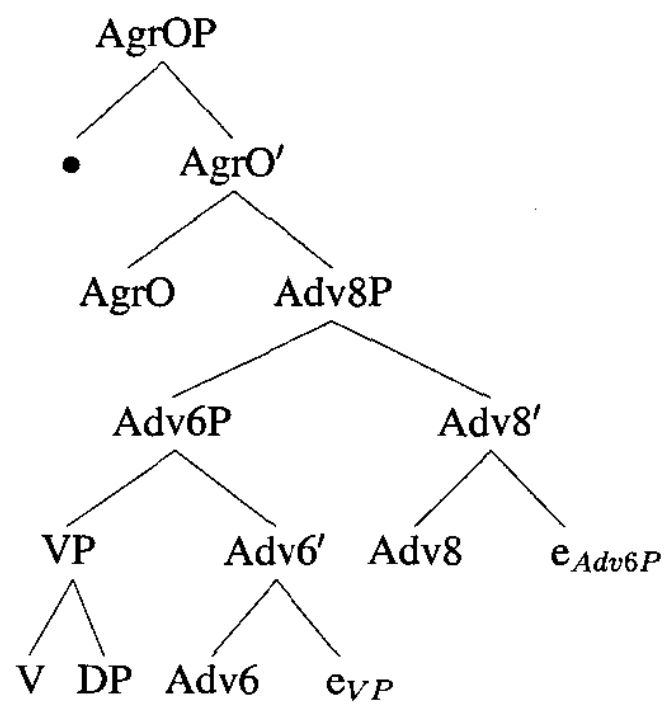

Example (20), however, presents us with a potential problem: we have here extraction from a complex specifier, and given a long series of adverbs, the DP could be indefinitely deeply embedded. Suppose we permit unlimited extraction from complex specifiers. We are then in a quandry with respect to the focus extraction: the explanation for the inaccessibility of the DPs for extraction, except for the externalized one, was that they were in a specifier island. In the article R\&T do invoke the island-hood of the fronted XP: "The predicate-fronting analysis predicts that, since the subject is the only argument not contained in some kind of island, it should behave differently from the other arguments." [p.124] How could we differentiate between the two cases? (Pearson has a different explanation, see below).

A potential solution comes from the former version of this analysis, namely the version in Rackowski (1998); here she adopts a strict Kaynean phrase structure with only one specifier per projection and differenties between categories and segments. Under these assumptions her structure for (20) was

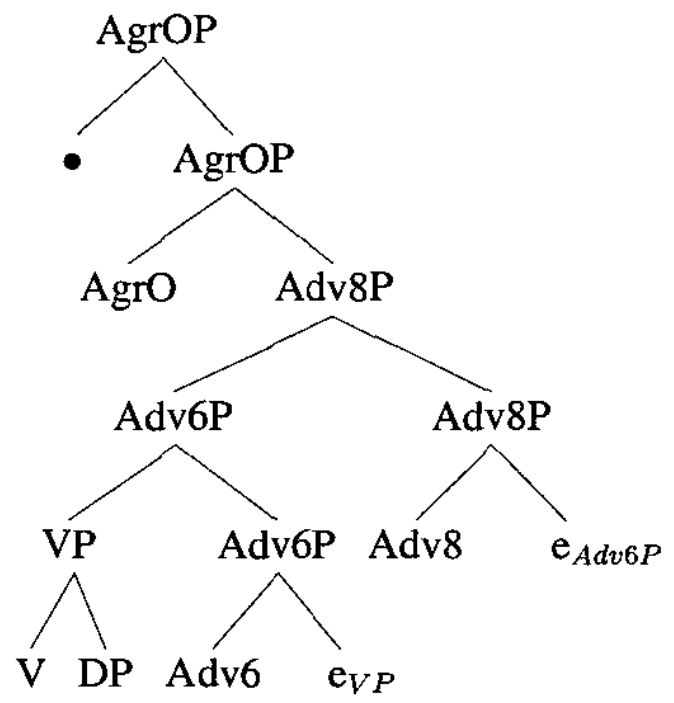

In (21) the only category which dominates DP is VP; the rest of the nodes are only segments. In traditional terms (i.e., Barriers), as Rackowski notes, there is nothing to block the properly governed DP from being extracted.

We then need to ask if this explanation is sufficient to nevertheless block extraction in, say, the focus or topic constructions. This would seem to be 
the case. Let us look at a "complete" derivation, much simplified. Note the position of DP in the Spec under VP $(22 a) \approx(21)$ from which it moves to AgrOP (22b). If TP subsequently moves (22c), the DP is then dominated by the category $\mathrm{AP}_{z}$ and all cateogries above $\mathrm{AP}_{z}$, freezing it in place (na (dia)...aza translates roughly as 'even...though'):

a.

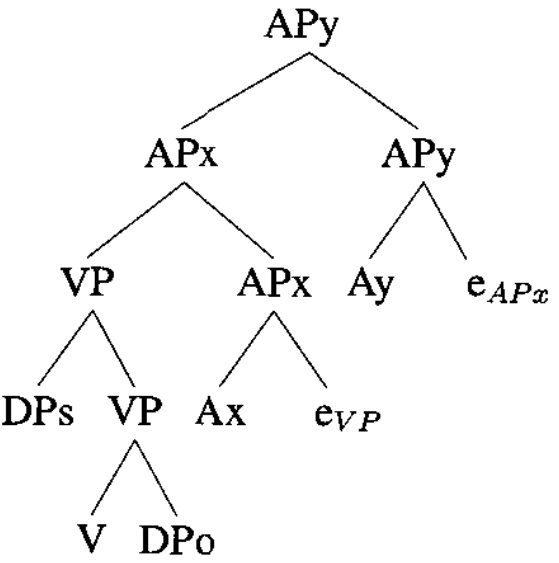

b.

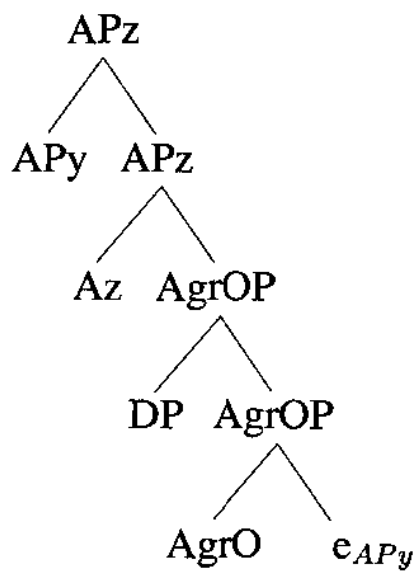

c. (tree on next page)

While this is a very neat solution, it depends on the segment/category distinction, which does not seem to be invoked in later work by Travis (2004), which uses the traditional X-bar theory. It also depends upon Barriers-style extraction restrictions and it remains to be seen whether we can translate this into a "Phase" explanation; see Thiersch (in prep.b).

We note that this approach is explicity rejected by Pearson, who claims to need multiple specifiers in his analysis, redefining C-command derivationally along the lines of Epstein et al. (1998) to achieve this. He links the extraction constraint to feature conflict similarly to the exclusion of simultaneous WH and Topic movement in Germanic; cf. discussion at the end of $\S 3.4 .1$, p.133. 
$(22 \mathrm{c})$

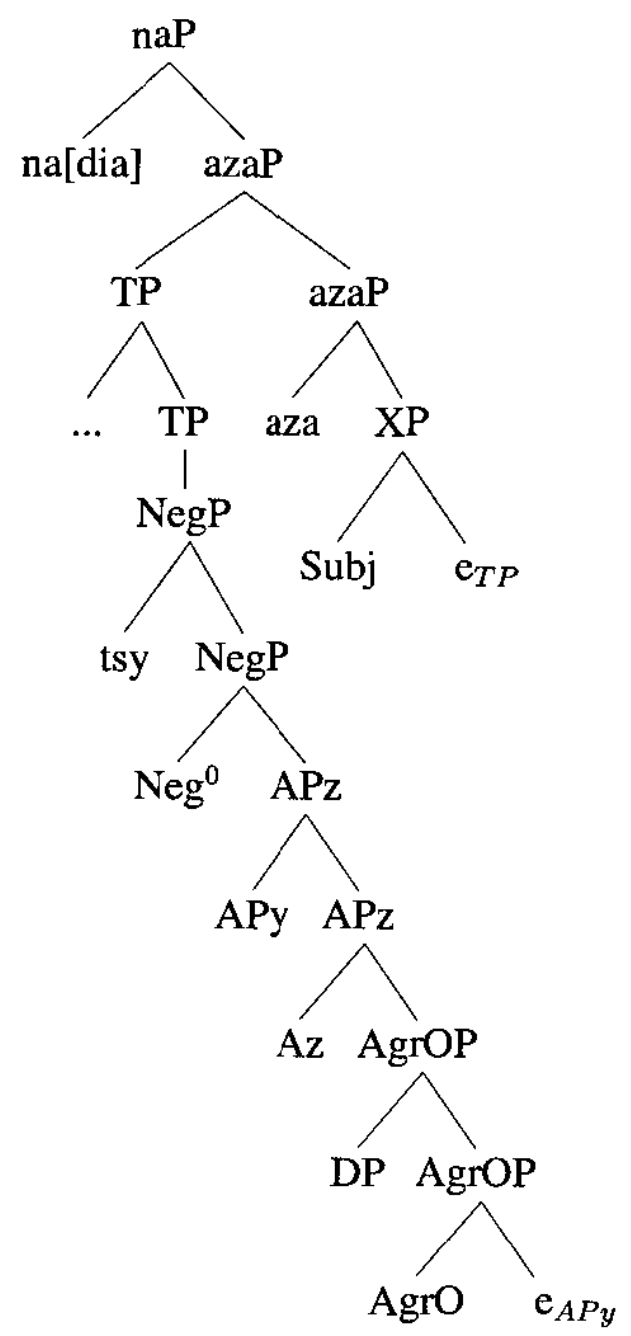

\section{The K\&Sz system}

While it is beyond the scope of this preliminary article to discuss the intricate system developed in K\&Sz for Hungarian and Dutch verb clusters (cf. Thiersch in prep.a), we would like to look briefly at their solution to the above problem, with an eye to a comparison of the issues involved. They are of course faced with the same problem as R\&T, namely the DP arguments associated with verbs have to be "gotten out" of the remnant constituents being rolled up. Indeed, since the data (verbal clusters) treated in $\mathrm{K} \& \mathrm{Sz}$ disregards adverbial constituents, the majority of the remnant character of their roll-up comes from repeated evacuation of an argument DP from a VP.

First of all, K\&Sz deal with extraction from complex specifiers by simply forbidding it by fiat. ${ }^{11}$

Glossing over many details (cf. Thiersch (in prep.a) the core of their system is as follows: although with five verbs the Hungarian verb cluster could theoretically have $5 !=120$ orders, in fact it has only four; e.g. with one (finite) true auxiliary, 2 semi-auxiliaries, a main verb and a VM (particle):
a. $1-2-3-5-4$
c. $1-5-4-3-2$
b. $1-2-5-4-3$
d. $5-1-2-3-4$

The verbs are base generated in the "English" (1-2-3-4-5) order following Kayne, the first pair $(\mathrm{V}+\mathrm{VM})$ must be inverted, and if the inversion stops, it cannot restart. Alternatively, the VM may raise (23d). Since they assume (i) 
only XP movement is involved, (ii) obligatory feature checking and (iii) no LF movement or counter-cyclic movement, the surface "English" order (23a) must also be derived. How do they prevent this from being circular, and how do they get the alternative orders with obligatory checking? The answer is optional pied-piping. We know pied-piping as a surface phenomenon is optional, so that if we encounter a structure like (24) [their $(72)=(79)$ ] where the $\mathrm{VP}^{+}$needs to move to Spec, $\mathrm{CP}(\bullet)$ to check a feature, it cannot (due to the prohibition of extraction from Spec), so we can choose either InflP ${ }^{+}$or PredP, pied-piping the rest.

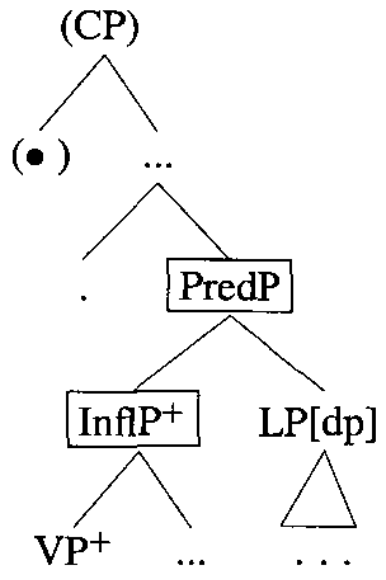

(An $\mathrm{XP}^{+}$is an extended projections based on Koopmans Generalized Doubly Filled Comp filter; cf. Thiersch (in prep.a).) This will eventually yield divergent derivations, giving the two orders.

Clearly this will run into problems if a projection contains a DP, just as in $R \& T$, and due to their prohibition on extraction from Spec, they do not have the escape-hatch of R\&T regardless of how we interpret their X-bar structure. Their solution is twofold:

a. They forbid "moving two constituents at the same time", by which they mean that in a structure like $\left[_{\mathrm{XP}} \ldots \mathrm{YP} \ldots \mathrm{ZP} \ldots \mathrm{]}\right.$, XP counts as two constituents under "certain circumstances"; i.a., when they are different projections, e.g., a VP containing a DP, and phonetically realized, but not when XP is the extended projection of, say, ZP.

b. They allow an arbitrary number of functional "pushing categories" (LPs, or licensing phrases) similar to AgrO, but with vacuous content.

Hence the DP must leave the VP at each step, due to (25a), and can leave due to (25b). (They note that this is not an entirely satisfactory solution.)

Of course we have not done justice to the complexity and subtlety of their system; but although they are able to generate the correct orders in Hungarian and parameterize the system for Dutch, there are numerous problems which they themselves note, and some they don't - e.g., many multiple derivations for the same string. Cf. Thiersch (in prep.a). The question arises as to whether they could relax the prohibition on extraction from specifiers, and use the Kaynean phrase structure to allow the DP to extract. Using very simplified structures we demonstrate that this seems unlikely. 
Supposed we start with the base structure V Prt DP, e.g., "take apart the radio+ACC" in (26a) ${ }^{12}$ and move the PrtP (in their terminology VM-phrase) to the Spec, $\mathrm{VP}_{4}$ as in (26b). We now have two basic options: if we assume (as opposed to $\mathrm{K} \& \mathrm{Sz}$ ), that a V can select a VP complement (or the infinitival functional projection thereof), we get a structure like (26c).

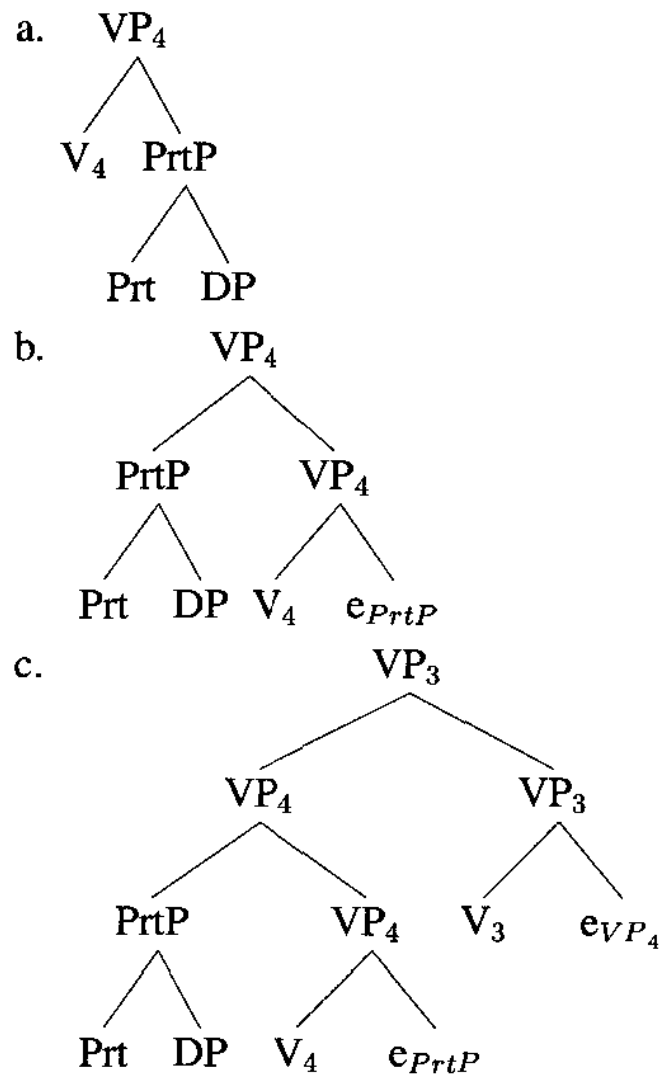

Here we see a structure akin to R\&T, and we observe that DP can leave later (it is not too deeply embedded) and the DP must leave at some time (because of checking features). (It is not clear what prevents it from leaving too early and becoming embedded in the verb-cluster, which is not permitted in Hungarian, as opposed to West Flemish and Swiss German, for example.) However, we are now faced with a different problem, namely what prevents the inverted cluster from unraveling? In K\&Sz, where the V selects an infinitival CP complement ${ }^{13}$ the inverted cluster becomes too deeply embedded in a specifier and cannot be undone; in (26c) nothing prevents the category $\mathrm{VP}_{4}$ from moving up to the specifier of $\mathrm{a} \mathrm{V}_{2}$, as in (27) [next page] for example, eventually generating the ungrammatical order $1+5+4+2+3$.

Similarly, what stops the roll-up? Roll-up in R\&T is stopped at a particular point, i.e., when the next specifier is filled. In K\&S it was stopped at any point where the verbs were in separate CPs and hence couldn't be moved together due to the "Move Only One Constituent" condition. But in (27) there is not enough structure to stop roll-up. Suppose we do allow the V to select a full $\mathrm{CP}$, as $\mathrm{K} \& \mathrm{Sz}$ do; then we have a structure like (28). But here if the CP moves up to $\times(\mathrm{Spec}, \mathrm{VP} 3)$ to create the inversion, the DP is now embedded to deeply in terms of categories to be extracted. Hence we are faced with a contradiction: we can't freeze verb complexes without freezing the DP. 
(27)

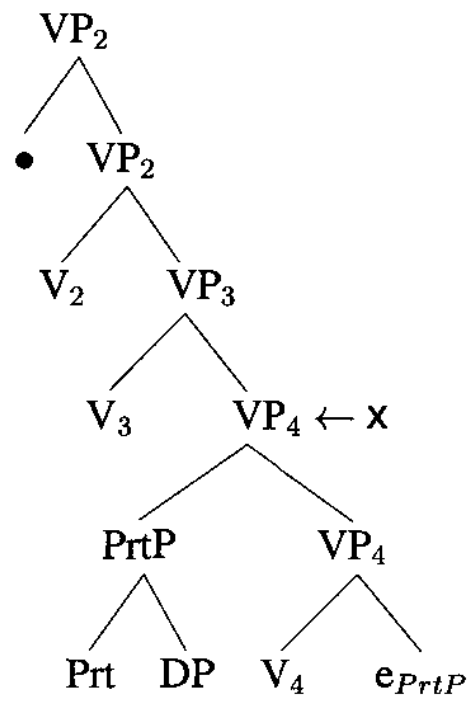

(28)

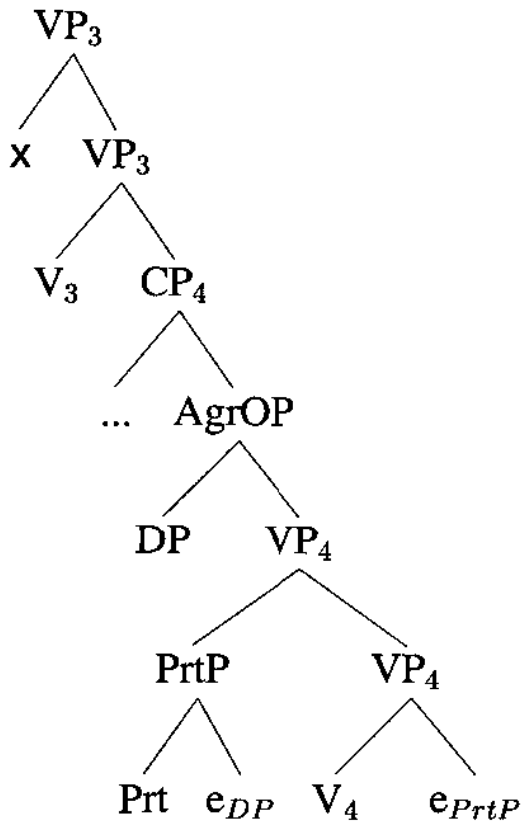

This hopefully reveals at least the tip of the iceberg regarding the extraction problem by comparing the technical details of these two systems: when invoking iterated RM the placement of arguments requires either arbitrarily deep extraction from a complex specifier or some system of local extraction; trying to use the R\&T system (albeit modified) in Hungarian seems unlikely to succeed. In the extended version of this article we discuss the problems with the reverse approach, applying a K\&Sz-like system to Malagasy, as well as looking at the $\mathrm{K} \& \mathrm{Sz}$ system in more detail.

\section{Endnotes}

0. ACKNOWLEDGEMENTS: Many thanks to colleages Hanitry Ny AlaGerull, Hans Broekhuis, Hans-Martin Gärtner, Paul Law, Jens Michaelis, Matt Pearson, Joachim Sabel, Lisa Travis, plus many more, for discussion and comments. As usual, the mistakes are the author's.

1. The analyses of $R \& T$ and Pearson are similar but differ in certain aspects. We return to this below. 
2. A possible example of extraction from a merged specifier could be sentences like those in Corver (1990) [his (152d), p.241]:

(i) [Hoeveel dagen $]_{i}$ is deze melk [ $e_{i}$ langer $]$ houdbar dan die how-many days is this milk e.c. longer keepable than that yogurt]?

yogurt

There is some evidence that this structure is incorrect, and this only involves extraction of a simple specifier; cf. recent work by Corver discussed in Thiersch (in prep.b).

3. The criteria distinguishing the two are not absolute; e.g., due to the constructions he is investigating, Kayne doesn't iterate as extensively as K\&Sz; on the other hand, Dutch also has RM I, and Hungarian possibly as well [(i)b is K\&Sz's (19), p.39]:

(i) a. [ $e_{j}$ geslagen $]_{i}$ heeft hij [de hond $]_{j} e_{i}$ beaten has he the dog

b. [Mutogatni $\left.e_{i} e_{j}\right]_{k}$ fogja akarni [a játékot $]_{i}[\mathrm{a}$ show-INF will-3SG want-INF the toy-ACC the gyerekeknek $]_{j} e_{k}$ children-DAT

'He will want to show the toys to the children'

Note however that mutogatni in (28b) is in a preverbal position normally occupied by the VM (verbal marker, like Dutch and German particles), and there is some controversy as to whether the structure shown (K\&Sz's) is correct or whether it is a case of head-movement. Hungarian lacks, as opposed to Dutch and German, the ability to displace the VP as a whole:

(ii) a. Den Kindern die Spielzeuge zeigen will er nicht. the-DAT children the-ACC toys show wants he not

b. * [ [vP Mutogatni a játékot a gyerekeknek] nem fogja akarni show the toys the children not will want

Hence although it has leftward scrambling/displacement of DPs, it lacks at least one of the operations to qualify for RM-I.

4. Note this is not deterministic for some "oblique" voices. See discussion in Al-Gerull (2004).

5. Pearson notes that the voice markers are really like Case, rather than being $\theta$-related, and are idiosyncratically governed by the verb. For example, one has to learn that write "governs" the dative (as opposed to IE accusative) [his (24)]:

(i) a. Nanoratra ny taratasy ny mpianatra

Pst-NomP.write Det letter Det student

'The student was writing the letter' 


\section{b. Nosoratan'ny mpianatra ny taratasy \\ Pst-DatP.write-Det student Det letter \\ 'The student wrote the letter'}

6. We ignore certain problems with Circumlocutional voice; again, cf. AlGerull (2004).

7. Pearson, following Paul (1999) analyses them as clefts involving an empty operator [his Ch.3 (122b)]:

(i) $\left[{ }_{\mathrm{PredP}} \mathrm{Wh} / \mathrm{Foc}_{i}\right]\left[{ }_{\mathrm{WhP}} \mathrm{Op}_{i} \text { no }\left[{ }_{\mathrm{PivP}} \mathrm{t}_{i}\left[{ }_{\mathrm{TP}} \mathrm{V} \ldots \mathrm{t}_{i} \ldots\right]\right]\right]_{i}$.

8. We take this somewhat controversial assumption as a given for the purposes of this article, as our interest lies in the problems associated with extraction from specifiers. NB: Pre-verbal adverbs can sometimes occur in variable positions with different meaning due to scope: e.g., "Tsy mbola corresponds to 'not yet', while mbola tsy means 'still not'." Rackowski (1998), p.10. They can also appear after the external argument ("Nachfeld").

9. Their translation of (13a) seems a bit odd semantically. The example (13b), their (21b), seems more natural, but doesn't illustrate the relevant word order. I assume substituting instony for foana in (13a) in the ensuing discussion will not affect the grammaticality, since the order of the adverbials in the Cinque heirarchy is preserved.

10. L. Travis, p.c. Note the movement of DP to AgrOP is presumably obligatory, so it would have to be extracted anyway as in the problematical example discussed below.

11. "Regarding moveable constituents, we assume that the *complement of $X$, or the specifier of the *complement of $\mathrm{X}$, may move to [Spec,XP]. *Complement is the transitive closure of the complement relation. In other words, only full specifiers and full complements on their own projection line can extract; parts of specifiers cannot." [p.38]

12. Not the surface order, of course. Note that their structure, [VP take [PP apart] [DP radio ]], is impossible given their assumptions, as it is trinary branching. I've plausibly assumed that the DP is an argument of the Prt.

13. Ignoring the yet more complicated structure suggested in their Chap.6.

\section{References}

Ala-Gerull, Hanitry Ny (2004) "Das zirkustanzielle Voice" Lecture and handout, Feb. 10, 2004, ZAS, Berlin.

Cinque, Gugliemo (1999) Adverbs and functional heads; a cross linguistic perspective. NY: Oxford U. Press

Corver, Norbert (1990) The syntax of left branch extractions. Ph.D. Diss., U.Tilburg

Epstein, Samuel D., Erich M. Groat, Ruriko Kawashima \& Hisatsugu Kitahara (1998) A derivational approach to syntactic relations. NY: Oxford Univ. Press

Guilfoyle, Eithne, Henrietta Hung \& Lisa Travis (1992) "Spec of IP and spec of VP: two subjects in Austronesian languages" Natural Language and Liniguitic Theory 10: p.375-414. 
Kayne, Richard (1994) The antisymmetry of syntax. Cambridge, MA: MIT Press

— (1998) “Overt vs. Covert Movements.” Syntax 1:128-191.

Koopman, Hilda (1996) "The Spec Head configuration" in Garnet, E., \& Lee, F., (eds.) Syntax at sunset (UCLA working papers in syntax and semantics 1, Dept. of Ling., UCLA) Reprinted in Koopman (2000) The syntax of specifiers and heads London: Routledge.

Koopman, Hilda \& Anna Szabocsi (2000) Verbal complexes. Current studies in linguistics 34. Cambridge, MA: MIT Press

Müller, Gereon (2002) "Two types of remnant movement" in Dimensions of Movement. From Features to Remnants, Artemis Alexiadou, Elena Aganstopoulou, Sjef Barbiers \& Hans-Martin Gärtner (eds). (Proceedings of the 1999 Potsdam Workshop on Remnant Movement.) Amsterdam: John Benjamins.

Paul, Ileana (1999) Malagasy clause structure. Ph.D. diss., McGill Univ.

Pearson, Matt (2001) The clause structure of Malagasy: a minimalist approach. Ph.D. diss. Dept. of Linguistics, UCLA

Rackowski, Andrea (1998) "Malagasy Adverbs" in I. Paul (ed.) The structure of Malagasy, v.II. UCLA Working Papers in Linguistics.

Rackowski, Andrea \& Lisa Travis (2000) "V-initial languages: X or XP movement and adverbial placement" In The syntax of verb-initial languages, Andrew Carnie \& Ethne Guilefoyle (eds.), Oxford UP, p.117-141.

Sabel, Joachim (2003) "Wh-questions and extraction asymmetries" in Proceedings of the 8th Conference of the Austronesian Formal Linguistics Association (AFLA VIII), Norvin Richards \& Andrea Rackowski (eds.), MTT Working Papers in Linguistics 44, Dept. of Linguistics, MIT, p.304-319.

Thiersch, Craig (2002) "Remnant Movement: Interim Report 1", DFG Projekt STA 519/1-1.

- (in preparationa) "Koopman \& Szabolsci's Verbal complexes; a critical evaluation" Ms. U.Potsdam

- (in preparationb) "Extraction from complex specifiers and the structure of degree phrases" Ms. U.Potsdam

Travis, Lisa (2004) "VP-, D ${ }^{0}$ movement languages: Malagasy in wonderland" AFLA 2004 lecture and handout; see also this volume.

Craig Thiersch U. Tilburg, U .Potsdam

Wilgenstraat 45

NL-5000 LE Tilburg

C.L.THIERSCH@UVT.NL 